\title{
Morfología comparada de los pleópodos del macho de Rhynchocinetes typus (Caridea: Rhynchocinetidae)
}

\author{
Comparative morphology of pleopods of Rhynchocinetes typus \\ (Caridea: Rhynchocinetidae) male \\ Rubí M. Martínez ${ }^{1}$ y Enrique Dupré ${ }^{1}$ \\ ${ }^{1}$ Departamento de Biología Marina, Universidad Católica del Norte, \\ Casilla 117 Coquimbo, Chile \\ edupre@ucn.cl
}

\begin{abstract}
The morphological adaptation that the first pair of pleopods has experienced in the Caridea group, permits to characterize them as gonopods which are used for transferring spermatophores to the female during the mating. In order to know the function of the pleopods in the transfer of spermatophores during the mating of the rock shrimp Rhynchocinetes typus, it was necessary to determine the
\end{abstract}

morphology of the male pleopods. The analysis of the first pair of pleopods showed a different morphology compared to the subsequent pair of pleopods, and notable adaptative differences in comparison with the typical morphology of other Caridea. These results suggest that both pairs of pleopods are involved in the transfer of spermatophores to the female.

Key words: Gonopods, endopods, exopods, appendix masculina

\section{Introducción}

En crustáceos decápodos, los dos primeros pares de pleópodos masculinos usualmente presentan una morfología diferente a los pleópodos posteriores (Bauer 1986a). Debido a su localización justo detrás de los gonoporos, esta modificación ha sido considerada como gonopoditos en la transferencia de espermatóforos a la hembra (Zhang \& Lin 2004).

El nivel de complejidad de los gonopoditos varía entre las especies de decápodos y es una medida de la distancia filogenética de la condición ancestral de los decápodos (Bauer 1986a) en la cual el primer y segundo par de pleópodos son birrameo y natatorio, presentando sólo el segundo pleópodo un apéndice masculino (Bauer 1986a).

Comparados con el petasma de camarones peneidos y la compleja estructura de transferencia de espermatóforos en Brachyura, el grado de modificación en camarones carideos es a menor nivel y por lo tanto es más primitivo (Zhang \& Lin 2004). Estos machos se caracterizan por la presencia de cincinnuli sobre el endopodito del primer par de pleópodos y por el apéndice masculino (AM) (proceso espinoso), sobre el borde medio del endopodito de cada segundo de pleópodos (Bauer 2004).

El conocimiento de la morfología funcional de los apéndices involucrados en la transferencia de espermatóforos ha sido utilizado para mejorar la comprensión de la estrategia de apareamiento que presentan los machos de una especie. En Rhynchocinetes typus (Milne Edwards, 1837), aun cuando se conoce el sistema de apareamiento (Correa \& Thiel 2003) y la alta competencia de los machos por aparearse con las hembras receptivas, no se conoce la morfología de los pleópodos masculinos ni cuál de los apéndices participaría en la transferencia de espermatóforos a la hembra durante el apareamiento. El conocimiento de la morfología de los pleópodos, junto con la participación de cada uno de ellos en el apareamiento, completaría el conocimiento detallado sobre la reproducción de esta especie y a su vez aporta antecedentes para predecir el comportamiento reproductivo de otras especies en las cuales sólo se tenga conocimiento de la morfología de sus apéndices, además de conocer el nivel evolutivo de la especie (Bauer 1986a, 2004), ya que el reemplazo de setas plumosas por espinas, ha sido considerada como un carácter más evolucionado para la especie (Bauer 2004).

\section{Material y métodos}

Se capturaron ejemplares del camarón de roca $R$. typus, correspondientes a machos robustus (Correa et al. 2000), desde el submareal rocoso de la bahía La Herradura, Chile (2959’ S, 71²2’W) mediante buceo autónomo y una red de mano. Los ejemplares recolectados se llevaron al laboratorio y fueron depositados individualmente en acuarios de $40 \mathrm{~L}$ con flujo continuo de aire y agua de mar filtrada a $5 \mu \mathrm{m}$ a temperatura ambiente $\left(14,5-18^{\circ} \mathrm{C}\right)$ y fotoperiodo natural. 
Para conocer en detalle la morfología de los pleópodos masculinos, se utilizaron seis ejemplares entre 16 y 21 mm de longitud cefalotorácica. A tres de los ejemplares se les amputaron los cinco pares de pleópodos con una tijera incandescente para la cauterización inmediata. Los pleópodos fueron fijados en glutaraldehído al 3\% en agua de mar, deshidratados en alcoholes de gradación creciente y secados a punto crítico en un aparato Samdri A-180 y cubiertos con oro en una metaladora JEOL. Posteriormente, fueron analizados con un microscopio electrónico de barrido JEOL T-300. A otros tres machos se les extrajeron los dos primeros pares de pleópodos y se les fotografió con una cámara Canon ${ }^{\circledR}$ adosada a un microscopio Nikon ${ }^{\circledR}$.

Los endopoditos de los dos primeros pares de pleópodos masculinos son diferentes en tamaño, forma y setación a los pares posteriores, sin embargo, los exopoditos, de los cinco pares de pleópodos son similares, presentando una forma ovalada y setas plumosas a lo largo de todo su borde. El tercer, cuarto y quinto par de pleópodos son morfológicamente similares entre sí (Fig.
1), los cuales disminuyen de tamaño desde el tercero al quinto.

El primer par de pleópodos (Fig. 2) presenta un exopodito cuya longitud es aproximadamente 2,5 veces su ancho máximo en su base; los bordes interno y externo están cubiertos por setas. El endopodito es de mayor tamaño que el exopodito; su longitud y ancho máximo son respectivamente 1/3 y 1/2 mayor que el exopodito; tiene la forma de una hoja lanceolada cuyo borde interno distal es recto y desprovisto de setas y termina en una punta aguzada curvada hacia el interior (Fig. 2A, D); su longitud es 2,0 veces mas larga que su ancho máximo. El borde externo está desprovisto de setas; en cambio, el borde interno presenta cinco setas plumosas cortas en el tercio proximal y numerosas setas cortas en el tercio distal donde se inserta del apéndice interno (AI) (Fig. 2A). El AI es cónico, alargado (Fig. 2B) en cuya base y parte del borde interno del endopodito se encuentra una leve depresión cubierta por setas espinosas cortas, aserradas y curvas (Fig. 2C, E).

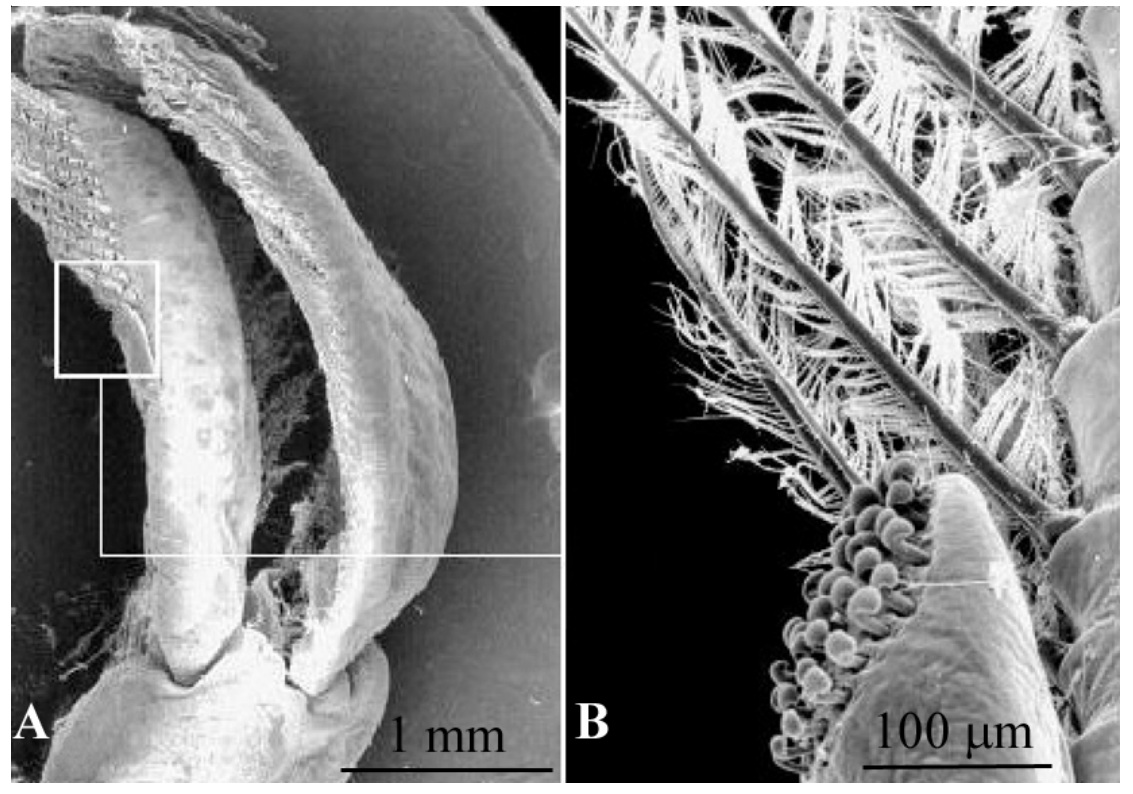

Figura 1

Quinto pleópodo izquierdo de R. typus, visto con microscopio electrónico de barrido. A) Pleópodo completo, B) Detalle del quinto pleópodo que muestra parte del apéndice interno con cincinnuli y algunas setas plumosas del borde interno del endopodito

Fifth left pleopod of $R$. typus shown by scanning electron microscopy. A) Entire pleopod.

B) Detail of the fifth pleopod showing the inner appendix with cincinnuli and plumose setae on the inner edge of endopodite 

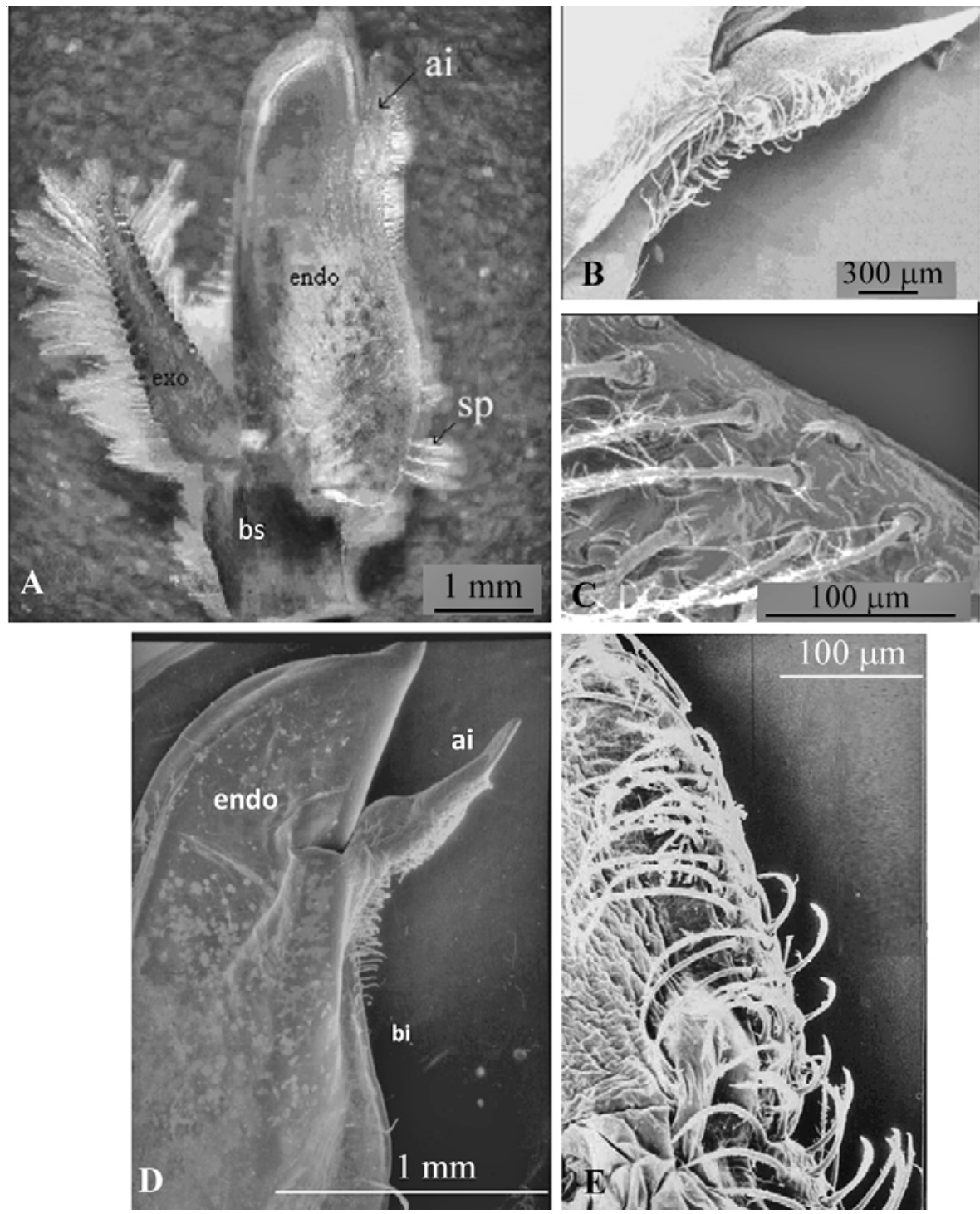

Figura 2

Primer par de pleópodos de R. typus. A) Cara anterior de pleópodo derecho visto con microscopia de luz, B) del apéndice interno del endopodito visto con microscopía electrónica de barrido (MEB), C) Espinas curvas presentes en el apéndice interno (MEB), D) endopodito y apéndice interno (MEB), E) borde interno de apéndice interno (MEB). Ai, apéndice interno; bi, borde interno; bs, basipodito; endo, endopodito; exo, exopodito; sp, setas plumosas

First pair of pleopods of R. typus. A) light microscopy of anterior face of the right pleopod, B) scanning electron microscopy (SEM) view of the inner appendix of endopod, C) SEM view of the spines on the inner appendix of endopod, D) SEM view of the endopod, E) SEM view of the inner border of appendix interna. Ai, appendix interna; bi, inner border; bs, basipodite; endo, endopodite; exo, exopodite; sp, plumose setae 


\section{Resultados y discusión}

El segundo par de pleópodos (Fig. 3) presenta un exopodito que es 1,3 veces más largo y 1/4 más ancho y de menor extensión que el endopodito, y su longitud total es 3,0 veces mayor que su ancho máximo. El endopodito, de menor tamaño que el exopodito (Fig. 3B), tiene una longitud de 3,5 veces su ancho máximo; en la mitad del borde interno se reinserta el apéndice interno (AI) y el apéndice masculino (AM). El AI, que es de menor tamaño que el AM, presenta en su extremo distal una superficie plana con gran cantidad de ganchos o cincinulis (Fig. 3C).

El AM, cuya longitud es 1/3 veces más largo que el AI, presenta espinas en el borde externo desde el tercio proximal en la superficie del extremo distal (Fig. 3B). Desde la parte media hacia arriba, el endopodito presenta espinas cortas por su superficie interna y espinas largas por el borde superior y en el tercio distal del borde externo, tomando un aspecto muy similar al AM (Fig. 3B).
Las espinas presentes en el borde externo del AM se insertan en forma perpendicular a la cara interna del endopodito, entrecruzándose perpendicularmente con las cortas espinas del endopodito (Fig. 3B). La mayor longitud del segundo pleópodo respecto al primer par, permite que ambos extremos distales se extiendan hasta el mismo punto en el esternito posterior torácico donde se encuentran los gonoporos.

Los resultados obtenidos, indican que los dos primeros pares de pleópodos de Rhynchocinetes typus difieren de los pares posteriores y presentan modificaciones respecto a la condición ancestral de decápodos, en la cual el primer pleópodo es birrameo y natatorio y el segundo similar con un apéndice masculino (Bauer 1986a), que es característica de la mayoría de las especies de camarones carideos (Zhang \& Ling 2004). El endopodito del pleópodo 1, difiere del pleópodo ancestral en que las setas plumosas son reemplazadas por cortas espinas aserradas y curvas (Fig. 4A). La presencia de setas espinosas en el borde interno del
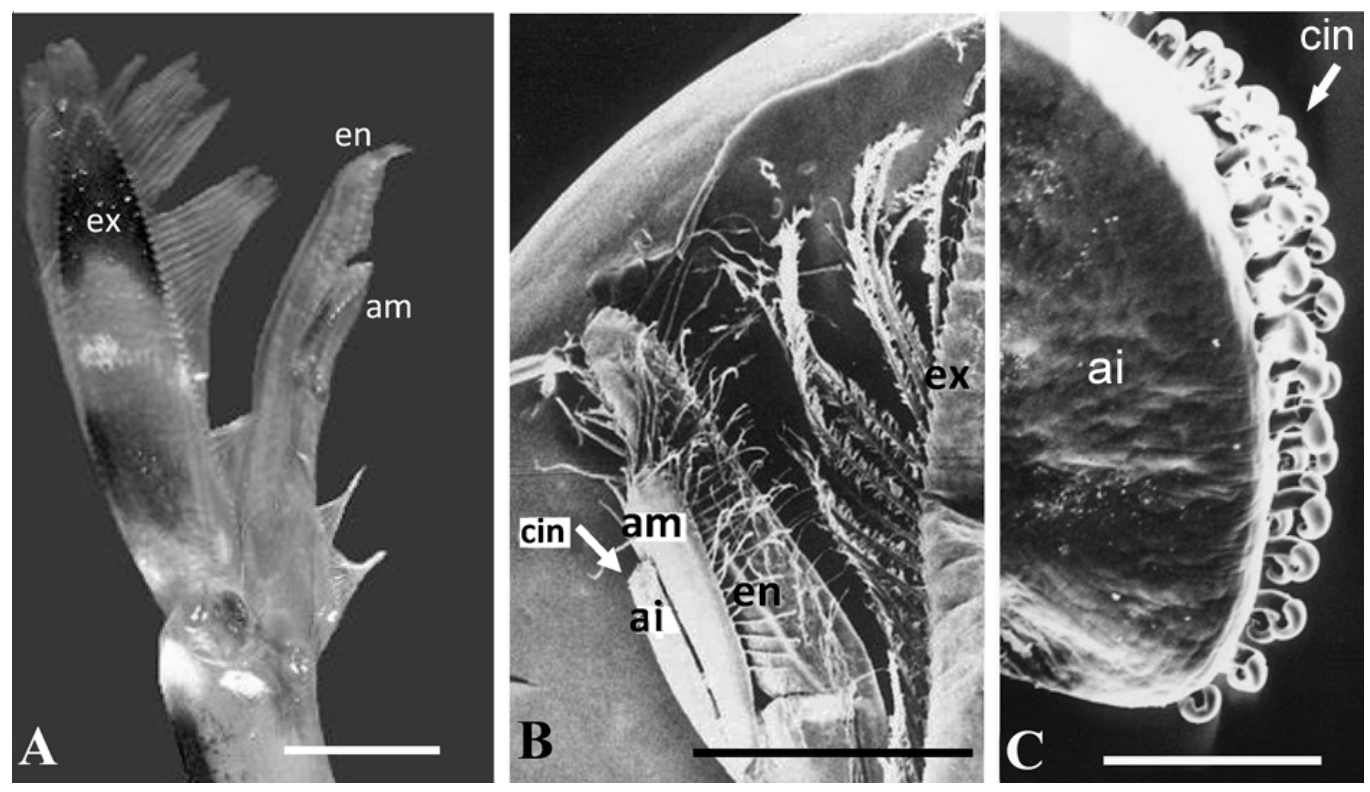

Figura 3

Segundo pleópodo derecho de $R$. typus. A) visto con microscopía de luz, B) visto con microscopía electrónica de barrido (MEB), C) extremo distal del apéndix interno visto con MEB; se observan cincinnuli (cin). Exopodito (ex); endopodito (en); apéndice interno (ai) y apéndice masculino (am). Barras $=100 \mu \mathrm{m}$

Second pair of pleopods of $R$. typus. A) light microscopy image, B) scanning electron microscopy (SEM) image, C) SEM image of the distal end of the appendix interna cincinnuli (cin). Exopodite (ex); endopodite (en); appendix interna (ai) and appendix masculina (am). Bars $=100 \mu \mathrm{m}$ 


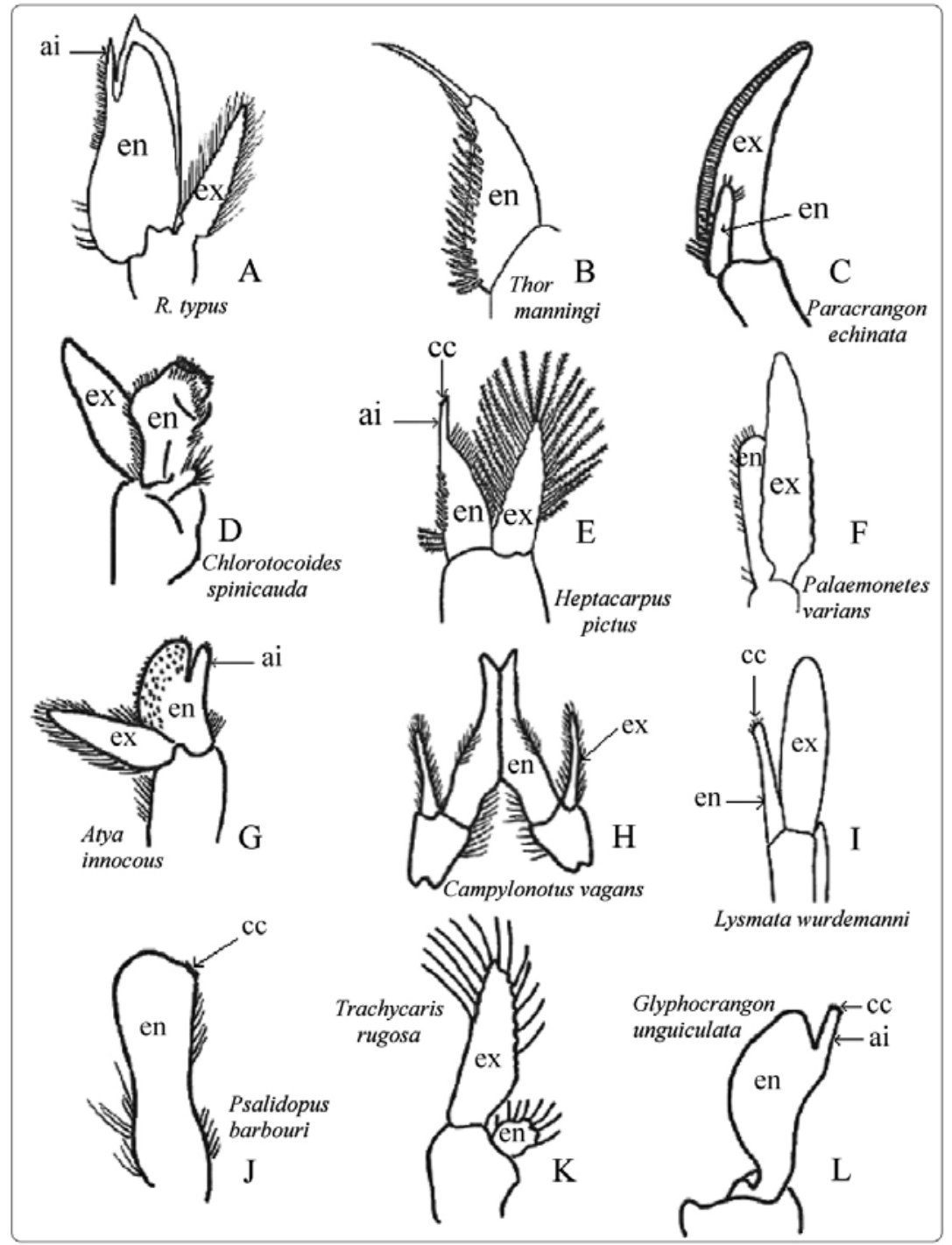

Figura 4

Primer par de pleópodos de $R$. typus comparado con los de otras especies del infraorden Caridea. A) Rhynchocinetes typus.

B) Thor manningi (Bauer 1986b). C) Paracrangon echinata (Komai \& Kim 2004). D) Chlorotocoides spinicauda (Bauer 2004). E) Heptacarpus pictus (Bauer 1976). F) Palaemonetes varians (González \& Cuesta 2006). G) Atya innocous

(Felgenhauer \& Abele 1982). H) Campylonotus vagans (Bauer 2004). I) Lysmata wurdemanni (Bauer \& Holt 1998). J) Psalidopus barbouri (Bauer 2004). K) Trachycaris rugosa (Criales 1992).

L) Glyphocrangon unguiculata (Bauer 2004). Ai, apéndice interno; cc, cincinnuli; en, endopodite; ex, exopodite

First pair of pleopods of $R$. typus compared with those of other species of caridean shrimp. A) Rhynchocinetes typus. B) Thor manningi (Bauer 1986b). C) Paracrangon echinata (Komai \& Kim 2004). D) Chlorotocoides spinicauda (Bauer 2004).

E) Heptacarpus pictus (Bauer 1976). F) Palaemonetes varians (González \& Cuesta 2006). G) Atya innocous

(Felgenhauer \& Abele 1982). H) Campylonotus vagans (Bauer 2004). I) Lysmata wurdemanni (Bauer \& Holt 1998). J) Psalidopus barbouri (Bauer 2004). K) Trachycaris rugosa (Criales 1992).

L) Glyphocrangon unguiculata. (Bauer 2004). Ai, appendix interna; cc, cincinnuli; en, endopodite; ex, exopodite 
endopodito, también ha sido registrada para otras especies de carideos tales como Thor manningi (Bauer 1986b), Paracrangon echinata (Komai \& Kim 2004), Chlorotocoides spinicauda (Bauer 2004), Heptacarpus pictus (Bauer 1976) y Palaemonetes varians (González \& Cuesta 2006) (Fig. 4B, C, D, E y F respectivamente). Atya innocous también presenta setas espinosas en el endopodito del primer par, pero a diferencia de las antes mencionadas, ellas se encuentran por la superficie interna de éste (Felgenhauer \& Abele 1982) (Fig. 4G). Esta modificación, de setas plumosas a espinosas ha sido considerada como un carácter más evolucionado (Bauer 2004).

Se observó la presencia de apéndice interno (AI) en el primer par de endopoditos de $R$. typus pero sin la presencia de cincinnuli, contrario a lo que ocurre en algunas especies como Campylonotus vagans en la cual no se observa apéndice interno en los endopoditos del primer par, ya que se encuentra fusionado al endopodito, pero se observan cincinnuli por todo su borde interno, lo que permite la unión de ambos endopoditos formando una estructura similar al petasma en peneidos (Bauer 2004) (Fig. 4H). Algo similar ocurre en Lysmata wurdemanni (Bauer \& Holt 1998) y Psalidopus barbouri (Bauer 2004), en las cuales también el AI se fusiona con el endopodito, pero los cincinnuli los presentan solo por el borde superior interno (Fig. 4 I, J respectivamente), lo que permite la unión distal de ambos endopoditos del primer par de pleópodos. En Thor manningi (Bauer 1986b), Chlorotocoides spinicauda (Bauer 2004), Paracrangon echinata (Komai \& Kim 2004), Trachycaris rugosa (Criales 1992) y Alpheus utriensis (Ramos \& von Prahl 1989) no hay rastro de apéndice interna ni cincinnuli. En las últimas dos mencionadas, el endopodito presenta un tamaño muy reducido en comparación con el exopodito (Fig. 5J, K respectivamente). Esto se ha observado en otras especies como Dioneus sandizelli y Salmoneus sp. (Dworschak et al. 2000). Glyphocrangon unguiculata no presenta setas plumosas ni espinosas en el endopodito, sólo un AI con cincinnuli en su parte distal (Bauer 2004) (Fig. 4 L).

Estos antecedentes permiten sugerir que las estructuras de los dos primeros pleópodos intervienen en el proceso de traspaso de espermatóforos a la hembra. Las setas cortas y curvas que cubren un pequeño canal en la base del apéndice interno del primer pleópodos de $R$. typus sugiere que esta conformación podría permitir la recepción de los espermatóforos extruídos desde el gonoporo para ser traslados hasta los pleópodos de la hembra durante los eventos de transferencia espermática (Correa et al. 2000). Los espermatóforos que tienen adhesividad quedarían enganchados en este apéndice interno y las setas de su base. Posteriormente podrían ser desenganchados o desprendidos por el apéndice masculino que posee setas largas en su extremo distal y borde externo para ser transferidos a la hembra. En esta transferencia podrían estar implicados los endopoditos del segundo par de pleópodos ya que sus setas de la parte superior interna del endopodito, las cuales al entrecruzarse con las setas del apéndice masculino ayudarían a desprender los espermatóforos para ser transferidos a la hembra. Otra estructura que podría ayudar a la transferencia es la presencia de cincinnulis en los apéndice interna del segundo pleópodo que permitiría la unión de ambos pleópodos para originar una corriente de agua suficientemente fuerte como para transferir los espermatóforos cuando se produzca el movimiento de ambos pleópodos. Todo este mecanismo sugerido podría dar cuenta de rápidas transferencias espermáticas como ocurre con el morfotipo typus de esta especie (Correa et al. 2000), los cuales además transfieren un mayor número de espermatóforos durante el apareamiento comparados con el morfotipo robustus (Correa et al. 2000).

Las especies Caridina bruneiana (Fig. 5C) (Choy 1992) y Atya innocous (Fig. 5D) (Felgenhauer \& Abele 1982) presentan un AM similar a $R$. typus. En la primera especie, Choy (1992) señaló la presencia de numerosas setas cortas en el borde distal e interno del AM, las cuales quedan posicionadas perpendicularmente sobre la superficie interna del AI del segundo par (Fig. 5C). En Atya innocous, el AM presenta numerosas espinas por todo el borde y también por la superficie interna (Fig. 5D). En Euryrhynchoides holthuisi, el AM se ha fusionado al reducido endopodito, formando una compleja estructura (ver Bauer 2004) (Fig. 5E). En algunas especies como Alpheus utriensis (Ramos \& von Prahl 1989), Palaemonetes turcuorum (González \& Cuesta 2006) Macrobrachium lamarrei y M. lanchesteri (Cai \& Ng 2002), el AM es distintivamente mas largo que el endopodito (Fig. 5F). En Procarididae, Synalpheus sp. (Alpheidae) (Bauer 2004) y Trachycaris rugosa (Hippolytidae) (Criales 1992) los machos no presentan AM ni AI (Fig. 5G).

Por otro lado, se determinó que todas las estructuras analizadas presentan la misma morfología en todos los individuos estudiados. No se encontraron estructuras intermedias o modificadas, lo que sugiere que no existiría protandria en esta especie.

Considerando la morfología de los dos primeros pares de pleópodos de Rhynchocinetes typus y los antecedentes antes mencionados, es muy probable que ambos apéndices estén involucrados en la transferencia de espermatóforos a la hembra durante el apareamiento. 


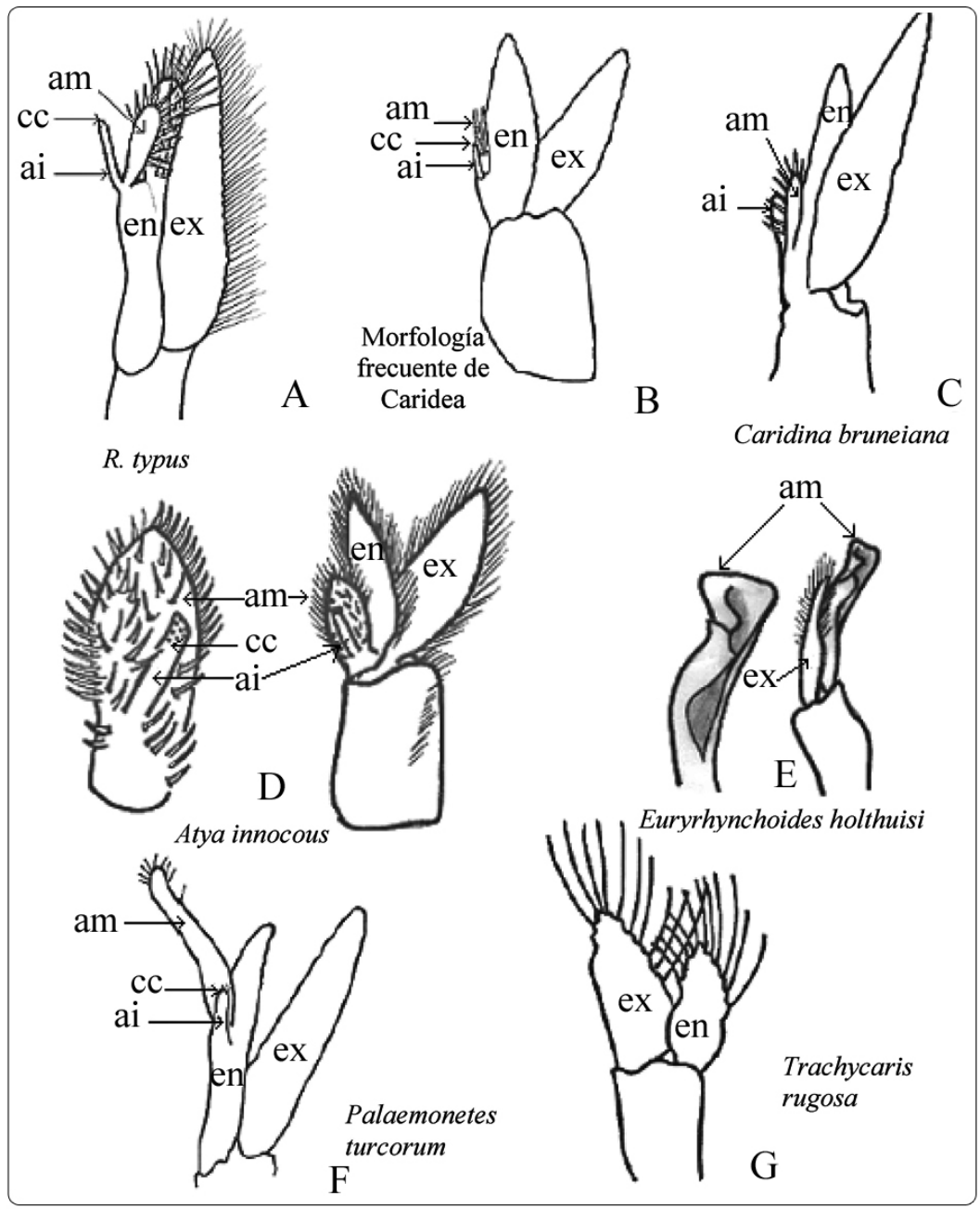

Figura 5

Segundo pleópodos de machos en especies del infraorden Caridea. A) Rhynchocinetes typus. B) Morfología característica de la mayoría de machos carideos (Bauer 2004). C) Caridina bruneiana (Choy 1992).

D) Atya innocous (Felgenhauer \& Abele 1982). E) Euryrhynchoides holthuisi (Bauer 2004). F) Palaemonetes turcorum (González \& Cuesta 2006). G) Trachycaris rugosa (Criales 1992). Ai, apéndice interno; am, apéndice masculino; cc, cincinnuli; en, endopodito; ex, exopodito

Second pair of pleopods of male caridean shrimp. A) Rhynchocinetes typus. B) Characteristic morphology of most Caridean males (Bauer 2004). C) Caridina bruneiana (Choy 1992). D) Atya innocous (Felgenhauer \& Abele 1982). E) Euryrhynchoides holthuisi (Bauer 2004). F) Palaemonetes turcorum (González \& Cuesta 2006). G) Trachycaris rugosa (Criales 1992). Ai, appendix interna; am, appendix masculina; cc, cincinnuli; en, endopodite; ex, exopodite 


\section{Agradecimientos}

Este estudio fue parcialmente financiado a través del proyecto FONDEF D05 I-10246 adjudicado al profesor Enrique Dupré.

\section{Literatura citada}

Bauer R. 1976. Mating behavior and spermatophore transfer in the shrimp Heptacarpus pictus (Stimpson) (Decapoda: Caridea: Hippolytidae). Journal of Natural History 10: 415440.

Bauer R. 1986a. Phylogenetic trends in sperm transfer and storage complexity in decapod crustaceans. Journal of Crustacean Biology 6: 313-325.

Bauer R. 1986b. Sex change and life history pattern in the shrimp Thor manningi (Decapoda: Caridea): a novel case of partial protandric hermaphroditism. The Biological Bulletin 170: 11-31.

Bauer R. 2004. Remarkable shrimps: adaptations and natural history of the carideans, $282 \mathrm{pp}$. University of Oklahoma Press, Oklahoma.

Bauer R \& G Holt. 1998. Simultaneous hermaphroditism in the marine shrimp Lysmata wurdemanni (Caridea: Hippolytidae): an undescribed sexual system in the decapod Crustacea. Marine Biology 132: 223-235.

Berg A \& P Sandifer. 1984. Mating behavior of the grass shrimp Palaemonetes pugio Holthuis (Decapoda: Caridea). Journal of Crustacean Biology 4: 417- 424.

Cai Y \& P Ng. 2002. The freshwater palaemonid prawns (Crustacea: Decapoda: Caridea) of Myanmar. Hydrobiologia 487: 59-83.

Choy S. 1992. Caridina bruneiana, a new species of freshwater shrimp (Decapoda, Caridea, Atyidae) from Negara Brunei Darussalam, Borneo. Zoologica Scripta 21: 49-55.

Correa C, J Baeza, E Dupré, I Hinojosa \& M Thiel. 2000. Mating behavior and fertilization success of three ontogenetic stages of male rock shrimp Rhynchocinetes typus (Decapoda: Caridea). Journal of Crustacean Biology 20: 628-640.
Criales M. 1992. Redescription of the hippolytid shrimp Trachycaris rugosa (Bate) (Crustacea: Decapoda: Caridea) from the western Atlantic, with notes on sexual dimorphism. Proceedings of the Biological Society of Washington 105(3): 562-570.

Dworschak P, A Anker \& D Abed-Navandi. 2000. A new genus and three new species of alpheids (Decapoda: Caridea) associated with thalassinids. Annalen des Naturhistorischen Museums in Wien 102: 301-320.

Felgenhauer B \& L Abele. 1982. Aspects of mating behavior in the tropical freshwater shrimp Atya innocous (Herbst). Biotropica 14(4): 296-300.

Gherardi F \& C Calloni. 1993. Protandrous hermaphroditism in the tropical shrimp Athanas indicus (Decapoda: Caridea), a symbiont of sea urchins. Journal of Crustacean Biology 13(4): 675-689.

González E \& J Cuesta. 2006. An illustrated key to species of Palaemon and Palaemonetes (Crustacea: Decapoda: Caridea) from European waters, including the alien species Palaemon macrodactylus. Journal of the Marine Biological Association of the United Kingdom 86: 93-102.

King M \& R Moffitt. 1984. The sexuality of tropical deepwater shrimps (Decapoda: Pandalidae). Journal of Crustacean Biology 4: 567-571.

Komai T \& J Kim. 2004. Shrimps of the crangonid genus Paracrangon Dana (Crustacea: Decapoda: Caridea) from the northwestern Pacific: taxonomic review and description of a new species from Japan. Scientia Marina 68(4): 511536.

Ramos G \& H von Prahl. 1989. Alpheus utriensis, new species (Caridea: Alpheidae), from Utria Sound, Pacific Coast of Colombia. Journal of Crustacean Biology 9(3): 477-481.

Sokolov V \& V Spiridonow. 2006. Development of external sexual characters of deep sea Bythocaris shrimps (Crustacea: Decapoda: Hippolytidae). Journal of the Marine Biological Association of the United Kingdom 86: 349359.

Zhang D \& J Lin. 2004. Fertilization success without anterior pleopods in Lysmata wurdemanni (Decapoda: Caridea), a protandric simultaneous hermaphrodite. Journal of Crustacean Biology 20: 470-473. 